\title{
Joint power and handoff control using a hybrid systems framework
}

\author{
Mehmet Akar and Urbashi Mitra \\ Communication Sciences Institute \\ Department of Electrical Engineering-Systems \\ The University of Southern California \\ Los Angeles, CA, 90089-2565 USA \\ E-mail: \{akar,ubli\}@usc.edu
}

\begin{abstract}
Power control and handoff are two significant problems for cellular wireless systems. While both problems have received considerable attention of late, the problems are not often treated in a joint manner. Combined downlink power control and handoff design for cellular communication systems using a hybrid system framework is considered herein. Two new algorithms are proposed. The first one is a hard handoff/power control algorithm that endeavors a tradeoff between three performance criteria: transmitted power, number of handoffs and call quality. The second algorithm is a joint soft handoff/power control algorithm that takes into account the effect of the number of base stations in the active set in addition to the above performance criteria. The significance of the algorithms is that they incorporate the effects of channel fading and mobility, and achieve a tradeoff between the satisfaction levels of the mobile user and the network operator, thereby provide satisfactory service for the user while reducing the burden on the network such as undesired switching between base stations. The tradeoffs involved in both algorithms are verified through simulations.
\end{abstract}

Keywords - Power control, handoff, resource allocation, cellular communication systems, hybrid systems, wireless

\section{INTRODUCTION}

Dynamic resource allocation in wireless communication systems is important to maintain a reliable communication link between a base station and a mobile user. In order to achieve this objective, the transmitted powers, base station assignments, and allocated channels may need to be updated as the mobile user moves in a cellular environment or when a new call is admitted to the network. Although these resources can be controlled individually, joint allocation improves capacity and battery life, and decreases interference [1]-[5].

Hanly [1] and Yates \& Huang [2] were the first to consider the problem of joint base station assignment and power control. They provided a decentralized combined power control/cell-site selection algorithm which minimized the total transmitted power and the total interference caused to other users [1]-[2]. An asynchronous version of the algorithm was also examined [2]. In [3], Wu and Bertsekas considered the same problem under the assumption that the control variables assumed values from a discrete set and obtained an algorithm based on integer programming methods. In [4], Papavassiliou and Tassiulas studied channel allocation jointly with base assignment and power control as a means to increase capacity, and proposed a cen- tralized heuristic algorithm, which tried to minimize the number of channels needed to accommodate all the calls. In [5], Rashid-Farrokhi et al. combined beamforming capabilities at the receiver with joint power control and cell assignment. However, the effects of user mobility and channel fading were not studied in any of the work discussed above.

This paper is focused on combined handoff and power control design for a mobile user in a fading environment. Handoff is the activity of changing the controlling base station whereas power control is used to regulate the transmitter power so that the carrier to interference plus noise ratio (CINR) at the receiver end is at a desired level. In our previous work [6]-[7], we developed an analytic framework with which we carried out both hard handoff design [6] and soft handoff design [7] under the assumption that the transmittal powers were already fixed. This paper will extend our previous work by incorporating power control in a joint decision that also involves handoff design, thereby take advantage of joint resource allocation.

Recall that the algorithm studied in [1]-[2] can be used for combined power control and cell-site selection. However, this algorithm is not robust to fluctuations in the received power level, and therefore may result in undesired switching in the network as explained below. Consider a network with a single mobile moving near the boundary of two neighboring cells. In this case, the combined power control and cell-site selection algorithm in [1]-[2] assigns the base station which needs to transmit the lowest amount of power to the mobile. However, it is clear that such a scheme will generate too many unnecessary handoffs when a small increase in the controlling base station power is still satisfactory to keep the existing link intact. This simple example illustrates a basic tradeoff between transmitted power and the number of handoffs. In this paper, our objective is to analyze this tradeoff in more detail.

We begin by modelling the combined handoff and power control process as a hybrid system in Section II. A hybrid system is a dynamical system that involves both discrete event and continuous state variables [8]. Based on this hybrid model, joint hard handoff design and power control is addressed in Section III and an optimization problem is set up to capture the tradeoff between call quality, average number of handoffs, and transmitted power. A suboptimal 
solution to this optimization problem leads to an implementable hard handoff/power control algorithm. Extension to joint soft handoff design and power control is carried out in Section IV by incorporating the average number of base stations in the active set into the optimization problem, and a combined soft handoff/power control algorithm is obtained as a suboptimal solution of the minimization problem. Further extensions in terms of uplink algorithms and incorporating co-channel interference are omitted due to space limitation (see [9] for incorporating interference). Finally, the algorithms are tested numerically in Section V and conclusions are given in Section VI.

\section{A Hybrid Model for Joint Power Control AND HANDOFF}

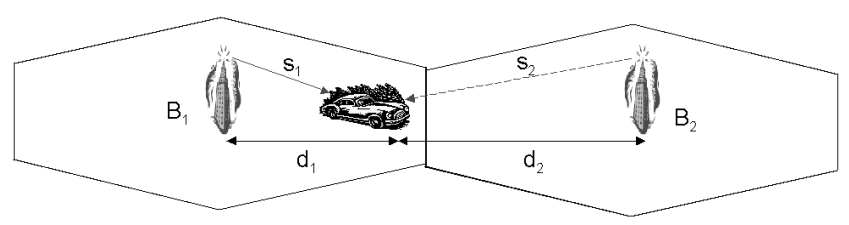

Fig. 1. Simulation scenario.

For simplicity of presentation, we will consider deriving combined power control and handoff algorithms for a mobile moving between two base stations, labelled $B_{1}$ and $B_{2}$; however, the ideas discussed in the sequel can be generalized to multiple mobiles and base stations. As shown in Fig. 1, it is assumed that the mobile is moving on a straight line with a constant velocity $v$ between the two base stations separated by $D$ meters from each other. Let $d_{i}(k)$, denote the distance between the mobile and base station $B_{i}, i=1,2$ at time instant $k$. The signals $s_{i}(k), i=1,2$ received by the mobile from base station $B_{i}$ at sampling instant $k$ can be written as

$$
s_{i}(k)=-\eta \log d_{i}(k)+u_{i}(k)+z_{i}(k), i=1,2
$$

where $z_{i}(k), i=1,2$ are zero mean Gaussian random processes that model the log-normal shadow fading and $u_{i}(k)$ are the transmitted power levels from $B_{i}$. The log-normal shadowing is assumed to have an exponential autocorrelation function, $\mathrm{E}\left[z_{i}(k) z_{i}(k+m)\right]=\sigma^{2} a^{|m|}$ where $\sigma^{2}$ is the variance of the shadow fading process, $a=e^{-d_{s} / d_{o}}$ is the correlation coefficient of the discrete-time fading process, $d_{s}$ is the sampling distance and $d_{0}$ is the correlation distance determining how fast the correlation decays with distance [10].

The measurements are further averaged using an exponential averaging window to alleviate the effect of shadow fading. The decay rate of the averaging window is determined by a constant $d_{a v}$ and after this filtering, the averaged signals $\bar{s}_{i}(k)$ are given by

$$
\bar{s}_{i}(k)=b \bar{s}_{i}(k-1)+(1-b) s_{i}(k), i=1,2
$$

where $b=e^{-d_{s} / d_{a v}}$. From (1) and (2), the state equation for the discrete-time part of the system is given by

$$
x_{i}(k+1)=f_{i}\left(x_{i}(k), u_{i}(k), z_{i}(k)\right), i=1,2
$$

where $x_{i}(k)=\left[d_{i}(k), s_{i}(k), \bar{s}_{i}(k)\right]^{T}, i=1,2$ and

$$
f_{i}\left(x_{i}, u_{i}, z_{i},\right)=\left[\begin{array}{l}
d_{i}(k)+(3-2 i) d_{s} \\
s_{i}(k+1) \\
b \bar{s}_{i}(k)+(1-b) s_{i}(k+1)
\end{array}\right] .
$$

Let $X_{i}(k) \in\{0,1\}, i=1,2$ denote the state variable describing whether the $i$ th base station is included in the active set for the mobile or not. We say that the $i$ th base station is in the active set at instant $k$ if $X_{i}(k)=1$ and otherwise if $X_{i}(k)=0$. We also assume a switching input $U_{i}(k) \in\{0,1\}, i=1,2$ to control the value of the state $X_{i}(k)$. If $U_{i}(k)=1$, the state is changed and if $U_{i}(k)=$ 0 , the current value of the state is preserved. Note that $X(k)=\left[X_{1}(k), X_{2}(k)\right]$ and $U(k)=\left[U_{1}(k), U_{2}(k)\right]$ are the state and the input of the discrete event part of the hybrid system which can be represented using the equation

$$
X_{i}(k+1)=F_{i}\left(X_{i}(k), U_{i}(k)\right), i=1,2
$$

where

$$
F_{i}\left(X_{i}(k), U_{i}(k)\right)=\left\{\begin{array}{l}
X_{i}(k), \text { if } U_{i}(k)=0 \\
0, \quad \text { if } U_{i}(k)=1 \text { and } X_{i}(k)=1 \\
1, \quad \text { if } U_{i}(k)=1 \text { and } X_{i}(k)=0
\end{array}\right.
$$

By definition, the discrete state, $X(k)$, can assume values from the finite set $\{[0,0],[1,0],[0,1],[1,1]\}$. However, in this paper, we will assume that the mobile user is connected to at least one base station at all times, eliminating the discrete mode $[0,0]$. This assumption is for mere convenience, and the state can be included to further accommodate call admission and call removal. When $X(k) \in \mathbb{X}_{s} \triangleq\{[1,0],[0,1],[1,1]\}$, both of the base stations can send signals to the mobile in the discrete mode $X(k)=[1,1]$, which is called the soft handoff mode [7]. If we further restrict the discrete state space by eliminating the soft handoff mode, (i.e. $\left.X(k) \in \mathbb{X}_{h} \triangleq\{[1,0],[0,1]\}\right)$, then at most one base station is allowed to communicate with the mobile at any time, and the communication link between the controlling base station and the mobile must be broken before establishing a new connection with another base station. This type of handoff is called hard handoff [6].

Both hard and soft handoff processes can be equivalently represented using finite automata consisting of two and three states, respectively, as shown in Fig. 2. In this figure, the transitions are marked in the input/output form $U_{1} U_{2} / X_{1} X_{2}$ where $U_{1}, U_{2}$ are the switching inputs that determine the transitions starting from the discrete state $X_{1}$ and $X_{2}$. For instance, in Fig. 2(b) the input $U_{1}=0, U_{2}=1$ forces a transition from the current state $X_{1}=1, X_{2}=0$ (and hence the notation 01/10) into the soft handoff mode $X=[1,1]$.

The joint power and handoff control problem is to control the switchings in Fig. 2 as well as to choose the transmitted power levels $u_{i}(k)$ so that the mobile user achieves satisfactory service without putting too much burden on the network and with minimum power. Thus, the objective of this paper is to choose the continuous inputs, 


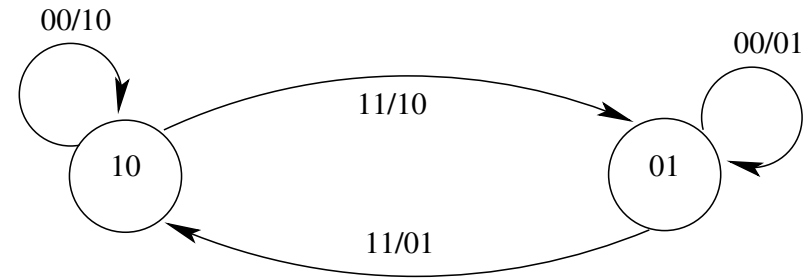

(a) Automata for hard handoff

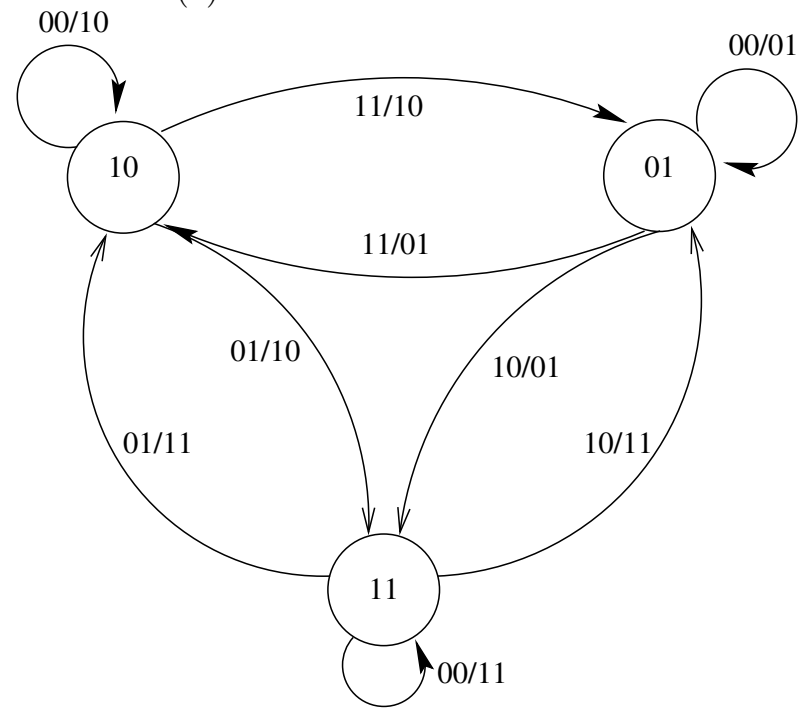

(b) Automata for soft handoff

Fig. 2. Models for hard and soft handoff

$u_{i}(k)$ and the switching inputs $U_{i}(k)$ at each instant $k$, $\left(1 \leq k<K=D / d_{s}\right)$ via some optimal or suboptimal method, which will be characterized through a cost function depending on the states and the inputs of the hybrid system model

$$
\left.\begin{array}{l}
x_{i}(k+1)=f_{i}\left(x_{i}(k), u_{i}(k), z_{i}(k)\right) \\
X_{i}(k+1)=F_{i}\left(X_{i}(k), U_{i}(k)\right)
\end{array}\right\} i=1,2 .
$$

\section{Power Control and Hard Handoff}

In this section, we will assume that only one base station is serving the mobile at all times, i.e., $X(k) \in \mathbb{X}_{h}$, and we intend to choose the inputs of the hybrid system to achieve a tradeoff between the following performance indicators:

$$
\begin{array}{ll}
N_{S D}: & \text { total number of signal degradations } \\
N_{h}: & \text { total number of handoffs } \\
P_{t}: & \text { total amount of power. }
\end{array}
$$

The indicator, $N_{S D}$, assesses the call quality for the mobile user, and the lower $N_{S D}$ is, the better the call quality is. We consider the number of handoffs as it is important to prevent excessive traffic and resource consumption in the network. Minimizing the total amount of power is important not only for maximizing the battery life, but also for decreasing the interference caused to other users in the network, and minimizing possible health hazards.

A signal degradation is defined to be the event that the averaged signal strength for the mobile falls below a thresh- old, $\Delta$, so $N_{S D}$ is expressed as

$$
N_{S D}=\sum_{k=1}^{K} \mathbf{1}_{\{\delta(\bar{s}(k), X(k))<\Delta\}}
$$

where $\mathbf{1}_{\{\cdot\}}$ is the indicator function and $\delta(\bar{s}(k), X(k))$ is given by

$$
\delta(\bar{s}(k), X(k))= \begin{cases}\bar{s}_{1}(k), & \text { if } X(k)=[1,0] \\ \bar{s}_{2}(k), & \text { if } X(k)=[0,1] .\end{cases}
$$

In the above expression for $\delta(\bar{s}(k), X(k))$, we have ignored the effect of possible co-channel interference. The effect of co-channel interference can be incorporated at the expense of increased computations, however we will omit these derivations due to space limitations [9].

Similar to the expression for $N_{S D}, N_{h}$ and $P_{t}$ are expressed as

$$
\begin{aligned}
N_{h} & =\sum_{k=1}^{K} \mathbf{1}_{\{X(k) \neq X(k-1)\}}, \\
P_{t} & =\sum_{k=1}^{K}\left[10^{u_{1}(k) / 10}+10^{u_{2}(k) / 10}\right] .
\end{aligned}
$$

Our objective is to derive an algorithm that endeavors to jointly minimize a weighted combination of the expected values of $N_{S D}, N_{h}$ and $P$. Thus, given the hybrid system in (5), our goal is to choose the continuous inputs, $u(1), u(2), \ldots, u(K)$ and the switching inputs, $U(1), U(2), \ldots, U(K-1)$, so that the best tradeoff between $E\left[N_{S D}\right], E\left[N_{h}\right]$ and $E\left[P_{t}\right]$ is achieved. This leads us to the following optimal control problem formulation.

Problem 1: Find $u=[u(1), u(2), \ldots, u(K)]$ and $U=$ $[U(1), U(2), \ldots, U(K-1)]$ which minimize the cost function

$$
\begin{aligned}
E\left[\sum_{k=1}^{K}[\right. & c \mathbf{1}_{\{X(k) \neq X(k-1)\}}+r_{1} 10^{u_{1}(k) / 10} \\
& \left.\left.+r_{2} 10^{u_{2}(k) / 10}+\mathbf{1}_{\{\delta(\bar{s}(k), X(k))<\Delta\}}\right]\right]
\end{aligned}
$$

subject to the system constraint in (5) and the power constraints $u_{i}(k) \leq u_{i, \max }, i=1,2$ where $c, r_{i}$ and $u_{i, \max }$ are constants with $r_{i}>0$ and $c \geq 0$.

The solution of the above problem can be obtained by arguments of dynamic programming. Unfortunately this solution will require the complete trajectory of the mobile in advance, making the algorithm impractical. By restricting the decision process to times $k$ and $k+1$ only, a suboptimal handoff algorithm can be derived. In this case, the decision making reduces to deciding whether to make a handoff at instant $k$ or not, and to choosing the transmitter power levels $u_{1}(k+1)$ and $u_{2}(k+1)$ for the next instant. The objective is then to determine $u(k+1)=\left[u_{1}(k+1), u_{2}(k+1)\right]^{T}$ and $U(k)$ so that

$$
\begin{array}{r}
P[\delta(\bar{s}(k+1), X(k+1))<\Delta]+c \mathbb{1}_{\{U(k)=[1,1]\}} \\
+r_{1} 10^{u_{1}(k+1) / 10}+r_{2} 10^{u_{2}(k+1) / 10}
\end{array}
$$


is minimized where $P[\delta(\bar{s}(k+1), X(k+1))<\Delta]$ is the probability of the event that the signal level for the mobile drops below the threshold $\Delta$, and it is equal to $Q\left(\frac{m_{s_{1}}(k)-\Delta}{\bar{\sigma}}\right)$ if $X(k)=[1,0]$, and $Q\left(\frac{m_{s_{2}}(k)-\Delta}{\bar{\sigma}}\right)$ if $X(k)=[0,1]$ with

$$
\begin{aligned}
m_{s_{i}}(k)= & b \bar{s}_{i}(k)+(1-b)\left[u_{i}(k+1)-a u_{i}(k)\right. \\
& +a s_{i}(k)-\eta \log \left[d_{i}(k+1) / d_{i}^{a}(k)\right] \\
\bar{\sigma}^{2}= & \left(1-a^{2}\right)(1-b)^{2} \sigma^{2} \\
Q(x)= & \frac{1}{\sqrt{2 \pi}} \int_{x}^{\infty} e^{-t^{2} / 2} d t
\end{aligned}
$$

If it exists, we define $u_{i, c}\left(u_{i}(k+1)=u_{i, c}\right)$ as the real root of the second order equation

$$
\frac{\ln 10}{10} u_{i}+\frac{\left(-\Delta+m_{s_{i}}(k)\right)^{2}}{2 \bar{\sigma}^{2}}-\ln \left(k_{i}\right)=0
$$

which minimizes the function

$$
Q\left(\frac{-\Delta+m_{s_{i}}(k)}{\bar{\sigma}}\right)+r_{i} 10^{u_{i}(k+1) / 10}
$$

where

$$
k_{i}=\frac{10(1-b)}{\sqrt{2 \pi} \bar{\sigma} \ln (10) r_{i}}, i=1,2 .
$$

We further let $u_{h i}^{\star}=\min \left(u_{i, c}, u_{i, \max }\right)$ and $J_{i}$ be the corresponding cost, i.e.,

$$
J_{i}=Q\left(\frac{-\Delta+m_{s_{i}}(k)}{\bar{\sigma}}\right)+r_{i} 10^{u_{h i}^{\star} / 10}, i=1,2
$$

where $m_{s_{i}}(k)$ is evaluated with $u_{i}(k+1)=u_{h i}^{\star}$. Then the suboptimal choice of the switching input is

$$
U^{\star}(k)= \begin{cases}{[1,1],} & \text { if } X(k)=[1,0] \text { and } J_{2}+c<J_{1} \\ {[0,0],} & \text { ot } X(k)=[0,1] \text { and } J_{1}+c<J_{2} \\ & \text { otherwise }\end{cases}
$$

and the continuous input is

$$
u^{\star}(k+1)=\left\{\begin{array}{l}
{\left[u_{h 1}^{\star},-\infty\right]^{T}, \text { if } X(k+1)=[1,0]} \\
{\left[-\infty, u_{h 2}^{\star}\right]^{T}, \text { if } X(k+1)=[0,1] .}
\end{array}\right.
$$

In this paper, (19) and (20) will characterize our joint hard handoff/power control algorithm. In particular, (19) corresponds to the hard handoff decision whereas (20) corresponds to power control. From (19), we note that a hard handoff decision is made (i.e., the controlling base station is switched, or $U(k)=[1,1])$, when the sum of expected cost of being served by the complementary base station and the cost of switching $(c)$ is lower than the expected cost of being served by the current controlling base station. As can be noted from (18), these expected costs depend on the expected signal quality and the expected transmitter power levels. Once the decision whether to handoff or not is made, the suboptimal downlink transmitted power levels are given by (20). Recall that the power levels are given in $\mathrm{dB}$, therefore the power value $-\infty$ corresponds to no transmission, e.g., $\left[u_{h 1}^{\star},-\infty\right]^{T}$ denotes $B_{1}$ transmitting with the power level $u_{h 1}^{\star}$ while $B_{2}$ does not transmit. The beauty of this algorithm is that it incorporates the effect of shadow fading, mobility and the cost of switching which further reduces undesired switching between the base stations (i.e., the ping-pong effect is mitigated). While doing so, a slight increase in transmitter power is used to keep the call quality at a satisfactory level.

\section{Power Control And Soft Handoff}

In this section, we relax the assumption in the previous section that only one base station can serve the mobile at any time, i.e., we let $X(k) \in \mathbb{X}_{s}$, and we intend to choose the inputs of the hybrid system to achieve a tradeoff between the satisfaction levels of the mobile user of interest and the network operator. Once again, call quality will be the important criterion for the mobile user. However, as far as the network operator is concerned, an additional cost criterion needs to be brought in to penalize the soft handoff mode. Recall that when $X(k) \in \mathbb{X}_{s}$, the mobile may be communicating with either of the base stations $B_{1}$ or $B_{2}$, or both. Straightforward intuition yields that the call quality for the mobile of interest will be best if it is serviced by both stations (i.e., $X(k)=[1,1])$. This is called macroscopic selection diversity. However, when $X(k)=[1,1]$, there is increased complexity in implementation and decreased network resources simply because there are more base stations that are transmitting signals. Obviously, this is undesirable unless the call for the mobile of interest is about to be lost. In this section, we characterize these cost functions explicitly and incorporate them in an optimization problem to design a joint handoff/power control algorithm.

We will still use the signal degradation criterion in (6) to characterize call quality for the mobile of interest. However, $\delta(\bar{s}(k), X(k))$ in (7) must be modified to accommodate the soft handoff mode, i.e., $\delta(\bar{s}(k), X(k))$ in $(7)$ is given by

$$
\delta(\bar{s}(k), X(k))=\left\{\begin{array}{l}
\bar{s}_{1}(k), \text { if } X(k)=[1,0] \\
\bar{s}_{2}(k), \text { if } X(k)=[0,1] \\
\max \left\{\bar{s}_{1}(k), \bar{s}_{2}(k)\right\}, \text { if } X(k)=[1,1]
\end{array}\right.
$$

where selective combining is used in the soft handoff mode to achieve diversity. Other combining techniques such as equal gain and any other weighted combining of the signals are also possible [7] (extended version). The call quality for the mobile of interest may be increased via three methods: (i) by increasing the transmitted power, (ii) by frequently updating the active set, or (iii) by employing the soft handoff mode. Associated with these three methods will be three performance criteria: Total power used $\left(P_{t}\right)$, total number of active set updates $\left(N_{u}\right)$, and total number of base stations in the active set $\left(N_{B}\right)$. The total power used $\left(P_{t}\right)$ for the downlink is still characterized by (9). Similarly, $N_{u}$ and $N_{B}$ can be expressed as

$$
N_{u}=\sum_{k=1}^{K} \mathbf{1}_{\{X(k) \neq X(k-1)\}},
$$




$$
N_{B}=K+\sum_{k=1}^{K} \mathbf{1}_{\{X(k)=[1,1]\}} .
$$

Note that $N_{u}$ replaces the total number of handoffs, which is considered in the hard handoff algorithm design in the previous section. Even though $N_{u}$ and $N_{h}$ have the same description, $N_{u}$ can conceptually be viewed as an extension of $N_{h}$ since the discrete state space is larger for the joint soft handoff and power control design. On the other hand, the total number of base stations in the active set $\left(N_{B}\right)$ is the additional criterion we will take into account in this section. Since we assume that the mobile is connected to at least one base station at each step, the constant $K$ (total number of steps from $B_{1}$ to $B_{2}$ ) is added to the expression for $N_{B}$. Considering this additional performance measure is important from the network operator's point of view in order to penalize unnecessary use of the soft handoff mode.

In this section we derive an algorithm that endeavors to jointly minimize a weighted combination of the expected values of $N_{S D}, N_{u}, N_{B}$ and $P$. Thus, given the hybrid system in (5), our goal is to choose the continuous inputs, $u(1), u(2), \ldots, u(K)$ and the switching inputs, $U(1), U(2), \ldots, U(K-1)$, so that the best tradeoff between $E\left[N_{S D}\right], E\left[N_{h}\right], E\left[N_{B}\right]$ and $E\left[P_{t}\right]$ is achieved. This leads us to the following optimal control problem formulation.

Problem 2: Find $u=[u(1), u(2), \ldots, u(K)]$ and $U=$ $[U(1), U(2), \ldots, U(K-1)]$ which minimize the cost function

$$
\begin{array}{r}
E\left[\sum _ { k = 1 } ^ { K } \left[c_{u}(k) \mathbf{1}_{\{X(k) \neq X(k-1)\}}+c_{b}(k) \mathbf{1}_{\{X(k)=[1,1]\}}\right.\right. \\
+r_{1}(k) 10^{u_{1}(k) / 10}+r_{2}(k) 10^{u_{2}(k) / 10} \\
\left.+\mathbb{1}_{\{\delta(\bar{s}(k), X(k))<\Delta\}}\right]
\end{array}
$$

subject to the system constraint in (5) and the power constraints $u_{i}(k) \leq u_{i, \max }$ where $c_{u}(k), c_{b}(k), r_{1}(k)$, and $r_{2}(k)$ are nonnegative tradeoff functions.

As in the previous section, the formulation of the above problem is such that its solution can be obtained using arguments of dynamic programming. Unfortunately, as before, such a solution requires the complete trajectory of the mobile in advance, which would make it impractical. By restricting the decision process to times $k$ and $k+1$ only, a suboptimal handoff algorithm can be obtained. In order to state this solution, let us assume that $r_{1}(k)=r_{1}>0$, $r_{2}(k)=r_{2}>0, c_{b}(k)=c_{b} \geq 0$, and the active set updates are penalized as

$$
c_{u}(k)= \begin{cases}c_{h}, & \text { if } U(k)=[1,1] \\ c_{s}, & \text { if } U(k) \in\{[0,1],[1,0]\}\end{cases}
$$

where $c_{h}$ and $c_{s}$ are nonnegative constants. The constants $c_{h}$ and $c_{s}$ correspond to the switching costs due to hard handoff $\left(c_{h}\right)$ and due to soft handoff $\left(c_{s}\right)$, respectively, and it is assumed that $c_{h} \geq c_{s}$.

The objective is to jointly determine whether to make a handoff (either hard or soft) or not, and determine the transmit powers, i.e., we want to choose $u(k+1)$ and $U(k)$ so that the cost function

$$
\begin{aligned}
& P[\delta(\bar{s}(k+1), X(k+1))<\Delta]+c_{b} \mathbf{1}_{\{X(k)=[1,1]\}} \\
& \quad+c_{u} \mathbf{1}_{\{U(k) \neq[0,0]\}}+r_{1} 10^{u_{1}(k+1) / 10}+r_{2} 10^{u_{2}(k+1) / 10}
\end{aligned}
$$

is minimized where $P[\delta(\bar{s}(k+1), X(k+1))<\Delta]$ can be computed as

$$
\begin{cases}Q\left(\frac{m_{s_{1}}(k)-\Delta}{\bar{\sigma}}\right), & \text { if } X(k)=[1,0] \\ Q\left(\frac{m_{s_{2}}(k)-\Delta}{\bar{\sigma}}\right), & \text { if } X(k)=[0,1] \\ Q\left(\frac{m_{s_{1}}(k)-\Delta}{\bar{\sigma}}\right) Q\left(\frac{m_{s_{2}}(k)-\Delta}{\bar{\sigma}}\right), & \text { if } X(k)=[1,1]\end{cases}
$$

and $m_{s_{i}}(k)$ is given by (12).

For the hard handoff modes, $X(k) \in\{[1,0],[0,1]\}$, the suboptimal power levels can be obtained as discussed in the previous section. Let $u_{h i}^{\star}$ be these power levels and $J_{i}$ be the corresponding cost values in (18). For the soft handoff mode, $X(k)=[1,1]$, the critical points for the suboptimal transmitted power levels can be obtained from the zeros of two coupled nonlinear equations

$$
\begin{aligned}
& \frac{\ln 10}{10} u_{1}+\frac{\left(-\Delta+m_{s_{1}}(k)\right)^{2}}{2 \bar{\sigma}^{2}} \\
& -\ln \left[k_{1} Q\left(\frac{-\Delta+m_{s_{2}}(k)}{\bar{\sigma}}\right)\right]=0 \\
& \frac{\ln 10}{10} u_{2}+\frac{\left(-\Delta+m_{s_{2}}(k)\right)^{2}}{2 \bar{\sigma}^{2}} \\
& -\ln \left[k_{2} Q\left(\frac{-\Delta+m_{s_{1}}(k)}{\bar{\sigma}}\right)\right]=0 .
\end{aligned}
$$

Among the stationary points of the above equations (which may correspond to a maximum or a minimum), let $u_{s 1}^{\star}$ and $u_{s 2}^{\star}\left(u_{1}(k+1)=u_{s 1}^{\star}\right.$ and $\left.u_{2}(k+1)=u_{s 2}^{\star}\right)$ be the ones which minimize the cost function

$$
\begin{aligned}
& Q\left(\frac{m_{s_{1}}(k)-\Delta}{\bar{\sigma}}\right) Q\left(\frac{m_{s_{2}}(k)-\Delta}{\bar{\sigma}}\right) \\
&+r_{1} 10^{u_{1}(k+1) / 10}+r_{2} 10^{u_{2}(k+1) / 10}+c_{b}
\end{aligned}
$$

and let $J_{12}$ be the corresponding value of the function. Then the suboptimal choice of the switching input is

$$
U^{\star}(k)=\left\{\begin{array}{cl}
{[1,1],} & \text { if } X(k)=[1,0] \text { and } \\
& J_{2}+c_{h}<\min \left\{J_{1}, J_{12}+c_{s}\right\} \\
& \text { or if } X(k)=[0,1] \text { and } \\
& J_{1}+c_{h}<\min \left\{J_{2}, J_{12}+c_{s}\right\} \\
{[1,0],} & \text { if } X(k)=[0,1] \text { and } \\
& J_{12}+c_{s}<\min \left\{J_{2}, J_{1}+c_{h}\right\} \\
& \text { or if } X(k)=[1,1] \text { and } \\
& J_{2}+c_{s}<\min \left\{J_{12}, J_{1}+c_{s}\right\} \\
{[0,1],} & \text { if } X(k)=[1,0] \text { and } \\
& J_{12}+c_{s}<\min \left\{J_{1}, J_{2}+c_{h}\right\} \\
& \text { or } X(k)=[1,1] \text { and } \\
& J_{1}+c_{s}<\min \left\{J_{12}, J_{2}+c_{s}\right\} \\
{[0,0],} & \text { otherwise }
\end{array}\right.
$$


and the continuous input is

$$
u^{\star}(k+1)=\left\{\begin{array}{l}
{\left[u_{h 1}^{\star},-\infty\right]^{T}, \text { if } X(k+1)=[1,0]} \\
{\left[-\infty, u_{h 2}^{\star}\right]^{T}, \text { if } X(k+1)=[0,1]} \\
{\left[u_{s 1}^{\star}, u_{s 2}^{\star}\right]^{T}, \text { if } X(k+1)=[1,1] .}
\end{array}\right.
$$

In this paper, (28) and (29) will characterize our combined soft handoff/power control algorithm. Note that this algorithm is a generalization of the combined hard handoff/power control algorithm given by (19) and (20) (This can be seen by choosing $c_{b}=\infty$, which implies that the soft handoff mode, $X(k)=[1,1]$, is never utilized, and therefore only hard handoff is possible). In particular, (28) corresponds to the handoff decision. In (28), the input selection $U(k)=[1,1]$ corresponds to hard handoff and the discrete inputs, $U(k)=[1,0]$ and $U(k)=[0,1]$, correspond to adding/dropping base stations, $B_{1}$ and $B_{2}$, to/from the active set, respectively (soft handoff). From (28), we note that switching from one discrete mode to another occurs only when the expected cost of being served in the candidate discrete mode plus the cost of switching to that mode is less than both the expected cost of being served in the current mode and the expected cost of being served in the third mode plus the cost of switching to the third mode. On the other hand, depending on which base stations are going to be in the active set, the suboptimal downlink transmitted power levels are given by (29), e.g., when only $B_{1}$ is to be transmitting, the power level is $u_{h, 1}^{\star}$, and in the soft handoff mode, the transmit powers are $u_{s 1}^{\star}$ and $u_{s 2}^{\star}$ for $B_{1}$ and $B_{2}$, respectively.

The implementation of the algorithm in (28) and (29) requires the computations of $u_{h i}^{\star}$ and $u_{s i}^{\star}, i=1,2$. The power levels $u_{h i}^{\star}, i=1,2$ can be directly computed as described in Section III. On the other hand, the candidate solutions for $u_{s i}^{\star}, i=1,2$ must be obtained from the zeros of (25) and (26), which does not seem to be analytically possible since these equations are coupled and highly nonlinear. Therefore, we will resort to nonlinear programming techniques [11]. In particular, we will use Newton's method to compute $u_{s 1}^{\star}$ and $u_{s 2}^{\star}$. To this end, let $p_{n}=\left[u_{s 1}(n), u_{s 2}(n)\right]^{T}$ where $u_{s i}(n)$ is an estimate for $u_{s i}^{\star}$, $i=1,2$ at the $n$-th iteration. The gradient descent type algorithm that will be used in this paper is given by

$$
p_{n+1}=p_{n}-\alpha_{n} D_{n} \nabla g\left(p_{n}\right)
$$

where $\alpha_{n}$ is the positive step size, $D_{n}$ is a positive definite matrix and $g\left(p_{n}\right)$ is the function given in $(27)$. The simple choice of $D_{n}=I$ leads to the steepest descent algorithm, however for faster convergence, we use $D_{n}=$ $\left(\nabla^{2} g\left(p_{n}\right)\right)^{-1}$ provided that $\left(\nabla^{2} g\left(p_{n}\right)\right)^{-1}$ is positive definite. If $\left(\nabla^{2} g\left(p_{n}\right)\right)^{-1}$ is not positive definite, then $D_{n}$ is taken to be $0.001 I$. There are also numerous ways of choosing the step size $\alpha_{n}$. In this paper, the step size is successively reduced according to the Armijo rule [11].

Using the boundedness properties of the function $g\left(p_{n}\right)$ (which can easily be derived from (27)), it can be proven that the sequence $p_{n}$ converges [11]. However, one setback is that the function (27) is not convex in $u_{1}$ and $u_{2}$, therefore any iterative method could converge to a local minimum. This implies that the initial guess for the iterative algorithm is crucial. A sample plot of the function in (27) for the values of $u_{1}, u_{2} \in\left(-\infty, u_{\max }=105\right]$ is shown in Fig. 3. It is clear from this plot that the cost criterion in (27) is bounded both from above and below. Furthermore, there is a region $\left[u_{1}^{\prime}, u_{\max }\right] \times\left[u_{2}^{\prime}, u_{\max }\right]$ in which the function seems to be convex. Therefore a good starting point for the algorithm is $u_{s i}=u_{\max }$, i.e., we initialize with the maximum power levels ${ }^{1}$. Although we have not managed to prove analytically that the algorithm converges to the global minimum starting from this initial condition, the desired results are always obtained in practice as will be demonstrated in Section V.

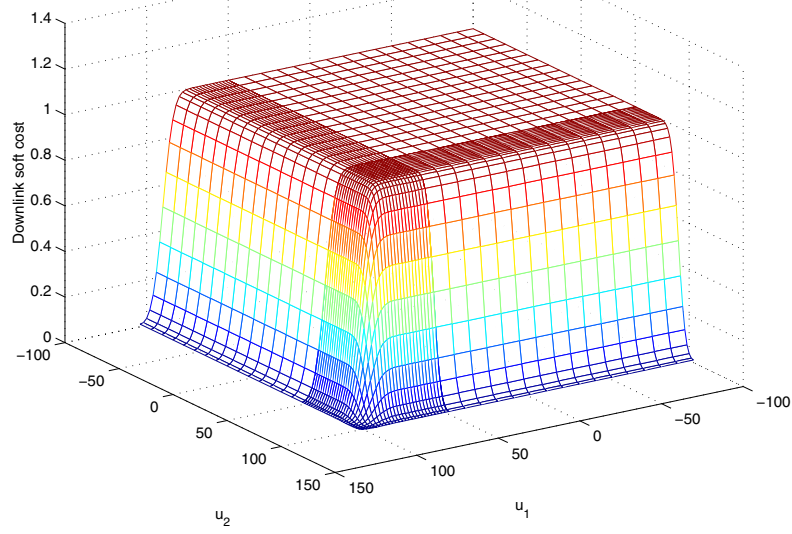

Fig. 3. Sample function for downlink soft cost criterion

\section{Numerical Results}

In this section, we present some numerical results for the algorithms in (19)-(20) and (28)-(29), respectively. The scenario in Fig. 1 is simulated as the mobile user moves from $B_{1}$ to $B_{2}$ for the specific simulation parameters given in Table 1 . The sampling distance $d_{s}=10 \mathrm{~m}$ corresponds to the speed of $72 \mathrm{~km} / \mathrm{hr}$ at a sampling period of $0.5 \mathrm{sec}-$ onds. The signal predictor $\hat{x}(k+1)=x(k)$ is used in the implementation of the algorithms.

We first look at the transmitted powers for both hard handoff modes $(X(k)=[1,0]$ and $X(k)=[0,1])$ and for the soft handoff mode $(X(k)=[1,1])$. Fig. 4 shows $u_{h i}^{\star}$ and $u_{s i}^{\star}, i=1,2$ as a function of the distance from $B_{1}$ under the assumption that distance information is known and there is no shadowing. An interesting observation is that $u_{s 1}^{\star}$ is equal to $u_{h 1}^{\star}$ (similarly for $u_{h 2}^{\star}$ and $u_{s 2}^{\star}$ ) till the mobile gets close to the boundary of the cells, then there is a neighborhood around the boundary in which the suboptimal soft power levels, $u_{s i}^{\star}, i=1,2$ are significantly lower than the suboptimal hard power levels, $u_{h i}^{\star}, i=1,2$.

\footnotetext{
${ }^{1}$ Another plausible starting point for the algorithm is $u_{s i}=u_{h i}^{\star}$, i.e., we set the initial point to be the suboptimal power levels for the hard handoff modes.
} 


\begin{tabular}{|l|l|}
\hline \hline$D=2000 \mathrm{~m}$ & base station separation \\
\hline$u_{\max }=105 \mathrm{~dB}$ & maximum allowable power \\
\hline$\eta=30 \mathrm{~dB}$ & path-loss exponent \\
\hline$\sigma^{2}=64 \mathrm{~dB}$ & variance of the fading process \\
\hline$d_{0}=30 \mathrm{~m}$ & correlation distance \\
\hline$d_{s}=10 \mathrm{~m}$ & sampling distance \\
\hline$d_{a v}=10 \mathrm{~m}$ & averaging distance \\
\hline$\Delta=0 \mathrm{~dB}$ & signal degradation threshold \\
\hline \hline
\end{tabular}

TABLE I

Simulation PARAMETERS

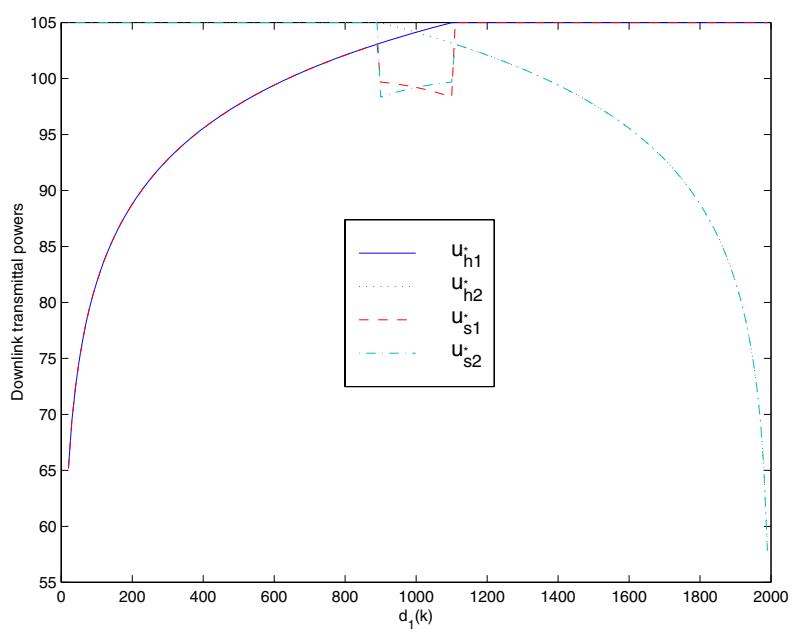

Fig. 4. Suboptimal downlink transmitted power levels.

Fig. 5 shows the simulation results for the combined hard handoff/power algorithm in (19) and (20) as the cost of switching $(c)$ and the weighting factor for the total transmitted power $\left(r_{1}=r_{2}=r\right)$ are varied, respectively. This graph clearly depicts the tradeoff between the three performance criteria of interest in the three dimensional space. Points C-F on this graph characterize the extremes for the algorithm, e.g., point $\mathrm{C}$ (high $r$ and high $c$ ) corresponds to low transmit power, low handoffs, which therefore result in more number of signal degradations and poorer call quality. On the other hand, point D is obtained by choosing a high value for $r$ and a low value for $c$. This choice of constants also leads to low power (because of high $r$ ), but a higher number of handoffs compared to point $\mathrm{C}$, because handoffs are not penalized as heavily. Although the transmit power is as low as for point $\mathrm{C}$, operating on point $\mathrm{D}$ leads to better call quality than point $\mathrm{C}$, simply because more handoffs are performed. Similar explanations can be made for points $\mathrm{E}$ and $\mathrm{F}$, where $r=0$.

Fig. 5 also shows another curve (from point A to point B). Point A on this curve corresponds to the algorithm proposed in [1]-[2], which minimizes the transmit power. Indeed, that algorithm results in a lower power choice. However, since the algorithm does not take the effect of shadowing into consideration, it leads to poorer call quality. Moreover it also leads to a high number of handoffs

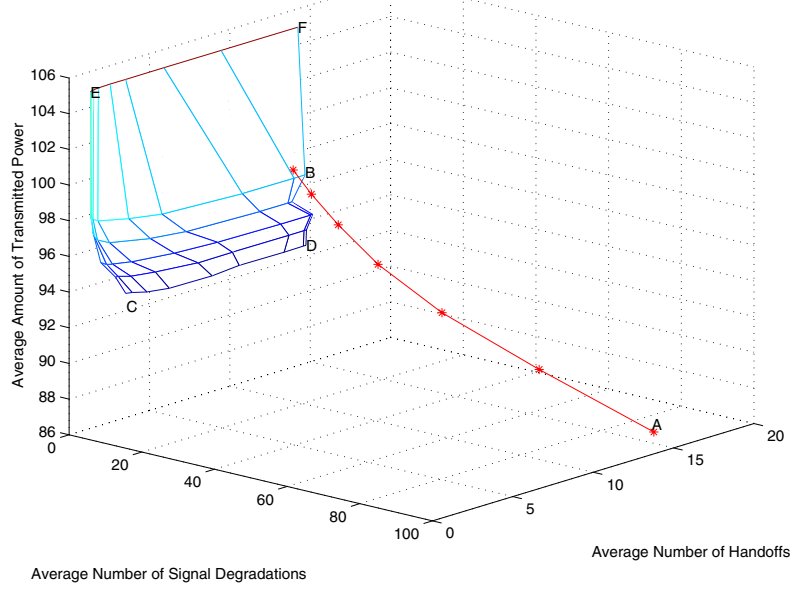

Fig. 5. Hard handoff performance criteria

since the cost of switching is not considered in the design of the algorithm. A modified version of this algorithm by varying $\Delta$ in the interval 0 to $12 \mathrm{~dB}$ (points $\mathrm{A}$ and $\mathrm{B}$ correspond to $\Delta=0 \mathrm{~dB}$ and $\Delta=12 \mathrm{~dB}$, respectively) leads to much better call quality, but still lacks the control on the number of handoffs performed.

Finally, Figs. 6-9 shows the simulation results for the combined soft handoff/power algorithm in (28) and (29) as the cost of switching, $c_{s}\left(c_{h}=c_{s}\right)$, and the weights for the transmit powers, $r_{1}=r_{2}=r$, are varied. As $c_{s}$ is increased, the cost of switching becomes more expensive leading to a decreased number of active set updates (Fig. 8) and therefore, degraded call quality (Fig. 6). In order to minimize the degradation in call quality, soft handoff is utilized more extensively for lower $c_{s}$ (Fig. 7), and hard handoff combined with increased transmitted power is used for higher $c_{s}$ (Figs. 7 and 9).

On the other hand, increasing $r$ leads to lower transmit powers (Fig. 9), which is a major cause of poor call quality (Fig. 6). However, this adverse effect is mitigated by frequently updating the active set (Fig. 8) or by entering into the soft handoff mode (Fig. 7).

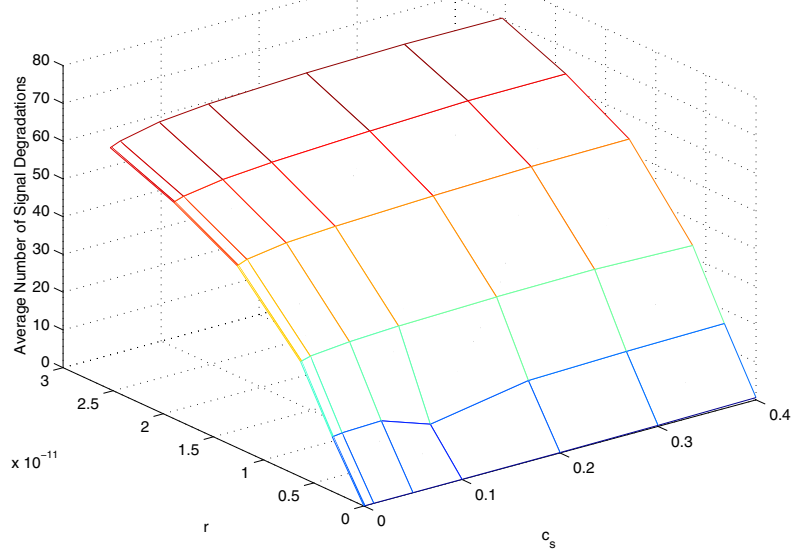

Fig. 6. Average number of signal degradations as $c_{s}$ and $r$ are varied 


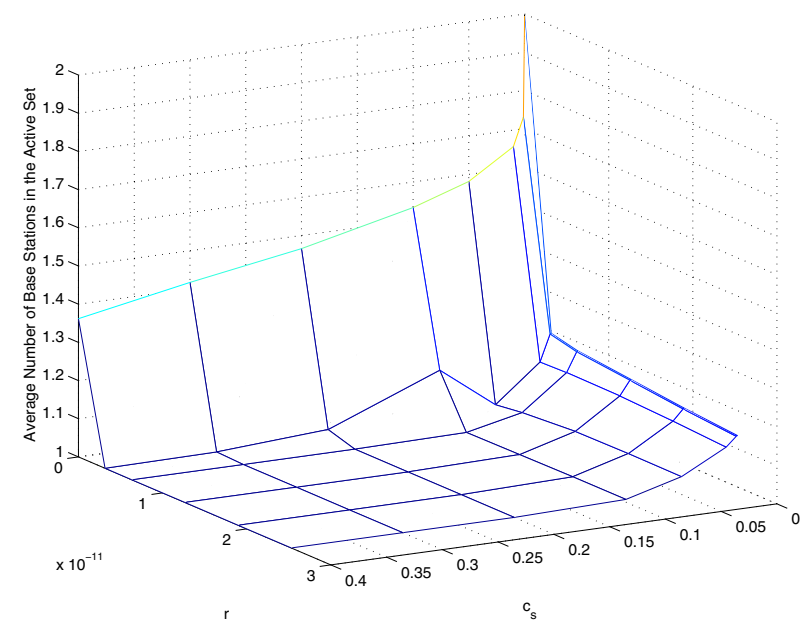

Fig. 7. Average number of base stations in the active set as $c_{s}$ and $r$ are varied

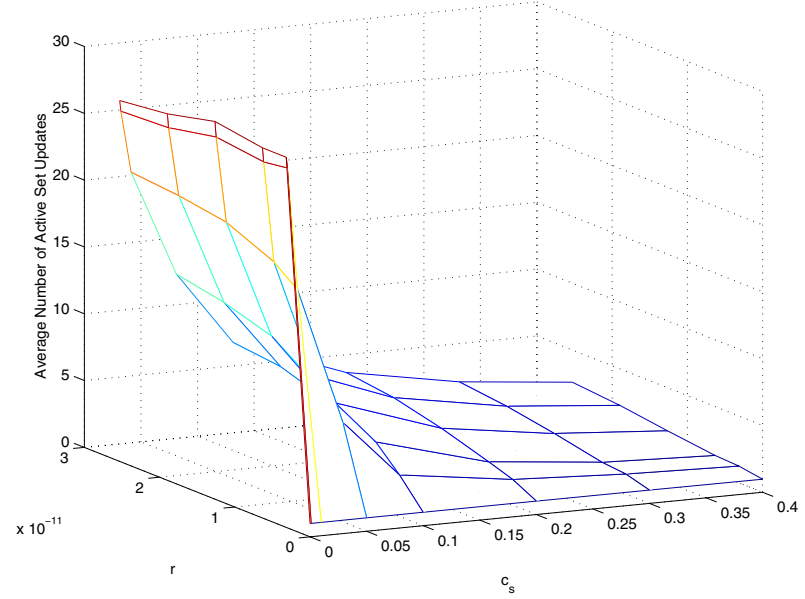

Fig. 8. Average number of active set updates as $c_{s}$ and $r$ are varied

\section{Conclusions}

In this paper, we have used a hybrid system model to represent combined downlink power control and handoff design. The continuous state part of the hybrid system corresponds to power control, and the discrete event part corresponds to handoff. Based on this hybrid model, various optimization problems are cast and two new algorithms are derived. These algorithms incorporate the effect of shadow fading and mobility, and achieve a satisfactory level of service for the mobile user without putting too much burden on the network. As such, the algorithms have the flexibility to incorporate online estimates of the parameters involved, such as the speed of the mobile. Furthermore, both of the algorithms can be modified to be used for uplink, and to deal with co-channel interference and multiple base stations, but such extensions are omitted in this paper due to space limitation. One open research topic is to show the convergence of the gradient descent algorithm used in Section IV to the global minimum of the function in (27).

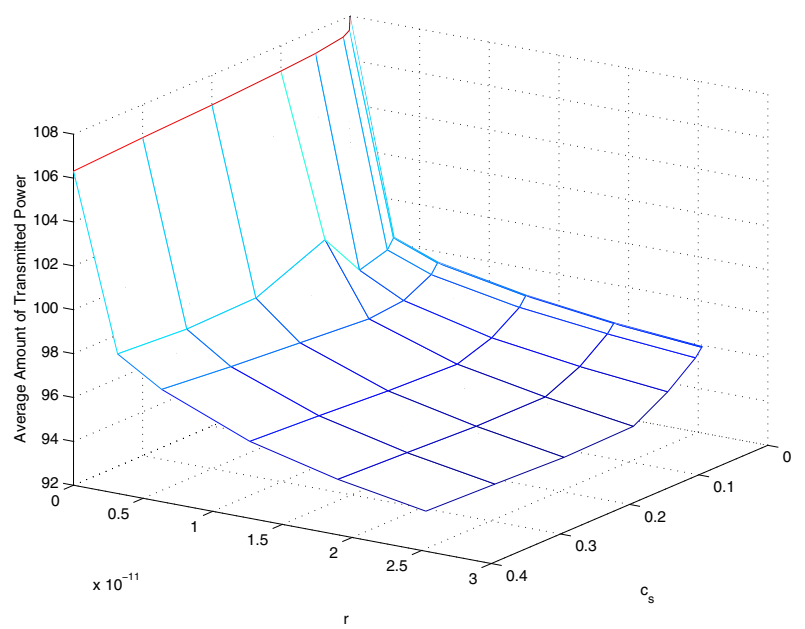

Fig. 9. Average amount of transmitted power as $c_{s}$ and $r$ are varied

\section{ACKNOWLEDGMEnT}

This research was supported by NSF grant ANI-0087761.

\section{REFERENCES}

[1] S. V. Hanly, "An algorithm for combined cell-site selection and power control to maximize cellular spread-spectrum capacity," IEEE Journal on Selected Areas in Communications, vol. 13, no. 7, pp. 1332-1340, September 1995.

[2] R. D. Yates and C. Y. Huang, "Integrated power control and base station assignment," IEEE Transactions on Vehicular Technology, vol. 44, no. 3, pp. 638-644, August 1995.

[3] C. C. Wu and D. P. Bertsekas, "Distributed power control algorithms for wireless networks," in Proc. Conference on Decision and Control, Phoenix, AZ, December 1999, pp. 3556-3561.

[4] S. Papavassiliou and L. Tassiulas, "Improving the capacity in wireless networks through integrated channel base station and power assignment," IEEE Transactions on Vehicular Technology, vol. 47, no. 2, pp. 417-427, May 1998.

[5] F. Rashid-Farrokhi, L. Tassiulas, and K. J. R. Liu, "Joint optimal power control and beamforming in wireless networks using antenna arrays," IEEE Transactions on Communications, vol. 46, no. 10, pp. 1313-1324, October 1998.

[6] M. Akar and U. Mitra, "Variations on optimal and suboptimal handoff control for wireless communication systems," IEEE Journal in Selected Areas of Communications, vol. 19, no. 6, pp. 1173-1185, June 2001.

[7] M. Akar and U. Mitra, "Design of soft handoff algorithms," in Proc. SPIE, Modeling and Design of Wireless Networks, Denver, CO, August 2001, vol. 4531, pp. 114-121 (Extended version submitted to IEEE Transactions on Wireless Communications).

[8] M. Akar, Variations on control of hybrid systems, Ph.D. thesis, Department of Electrical Engineering, The Ohio State University, Columbus, OH, 1999.

[9] M. Akar and U. Mitra, "Interference management in soft handoff algorithm design," in Proc. of Conference on Information Sciences and Systems, Princeton, NJ, March 2002.

[10] M. Gudmundson, "Correlation model for shadow fading in mobile radio systems," Electronic Letters, vol. 27, no. 23, pp. 21452146, November 1991.

[11] D. P. Bertsekas, Nonlinear Programming, Athena, Belmont, MA, 1999. 